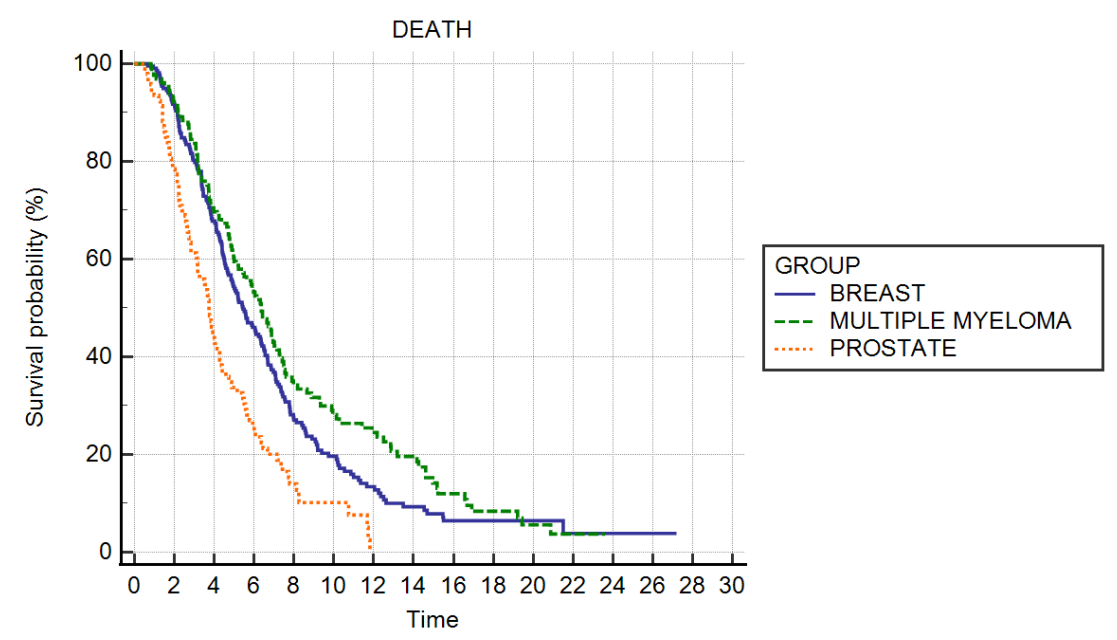

\title{
Expected survival of cancer and myeloma patients undergoing bisphosphonates or denosumab. Potential impact on diagnosis and management of Osteonecrosis of Jaws (ONJ)
}

Vittorio Fusco ${ }^{1}$, MARCO CABRAS $^{2}$, Alessio Gambino², Massimo DI MAIO ${ }^{2}$, Roberto Freilone ${ }^{3}$, Marco Tampellini ${ }^{4}$, Incoronata Romaniello ${ }^{5}$, Maria Rosa Alvaro ${ }^{6}$, Gianmauro Numico ${ }^{7}$, Maura Rossi ${ }^{1}$, Cinzia Ortega, Marcello Tucci $^{8}$, Davide Ottaviani ${ }^{9}$, LUCIO BUFFONI ${ }^{9}$, Giorgio Scagliotti ${ }^{2}$, Giorgio Vellani ${ }^{10}$, Paola Varese ${ }^{11}$, Mario Franchini ${ }^{12}$, claudia galassi ${ }^{13}$

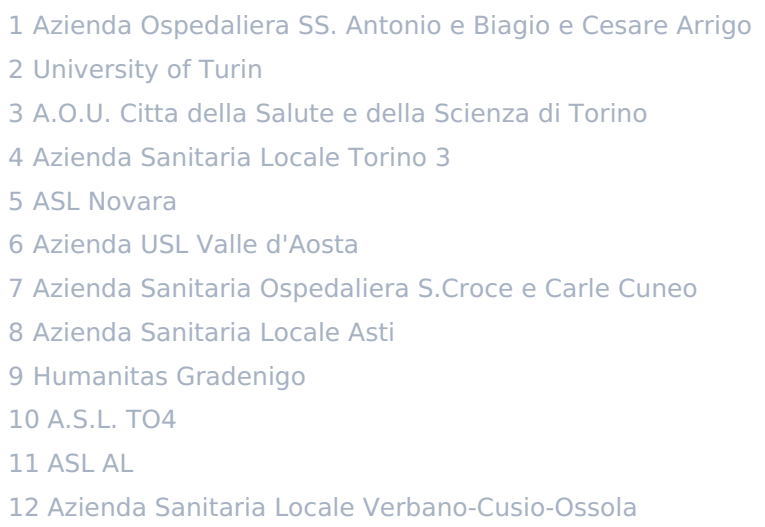

\section{Abstract}

Survival of cancer and myeloma patients after diagnosis of bone lesions is largely variable. Appropriate medical therapy together with antiresorptive treatment (bisphosphonates or denosumab) can obtain 
prolonged survival with adequate quality of life. Osteonecrosis of Jaws (ONJ) is not rare in patients with bone metastatic cancer and myeloma patients, occurring mostly after prolonged antiresorptive treatment. Expected survival can influence the choice of antiresorptive treatment, the perception of ONJ risk, and even the ONJ management. We reviewed survival data after start of antiresorptive treatment of 509 ONJ patients registered in a cancer network database (Rete Oncologica Piemonte-Valle d'Aosta, North-Western Italy).

Main characteristics: 196 males, 313 females; median age: 68 (39-89) years. Underlying disease: breast cancer: $42.9 \%$; myeloma: $25.3 \%$; prostate cancer: $17.2 \%$; lung cancer: $6.7 \%$; renal cancer: $2.5 \%$; other cancer types: $4.3 \%$. Main antiresorptive treatment: zoledronic acid: 79.4\%; denosumab: 5.3\%; other drugs/sequences: $15.3 \%$.

Actuarial median survival (range) after the start of antiresorptive treatment was 66.0 (95\% $\mathrm{Cl} 57-77$ ) months for breast cancer patients, 77.3 (95\% Cl 61.9-86.8) months for myeloma patients, 47.3. $(95 \% \mathrm{Cl}$ 37.8-51.9) months for prostate cancer patients, 29.4 (95\% Cl 20.4-40.1) months for lung cancer patients, 39.4 (95\% Cl 20-107.3) months for renal cancer patients, 82.4 (95\% Cl 51.9-150.9) months for other cancer patients.

Two-, three- and four-year actuarial survival rates were respectively $91.7 \% \%, 80.2 \%, 67.4 \%$ for breast cancer patients, $91.4 \%, 83.7 \%, 68.9 \%$ for myeloma patients, $78.4 \%, 61.2 \%, 41.9 \%$ for prostate cancer patients; $55.8 \%, 32.3 \%, 23.5 \%$ for lung cancer patients; $61.5 \%, 53.8 \%, 46.1 \%$ for renal cancer patients; $81.8 \%, 77.3 \%, 68.1 \%$ for others cancer types.

Our data support careful evaluation of short and long-term actuarial ONJ risk (versus short-term absolute risk) in the choice of antiresorptive treatment duration for bone metastatic cancer and myeloma patients. Furthermore, exclusion of jawbone surgery due to expected short survival in most of ONJ patients seems not warranted.

Background: Expected survival of patients with bone metastases of solid tumors (breast, prostate, lung, renal cancer, etc) [1][2][3] and of myeloma patients ${ }^{[4][5]}$ is largely variable in "real life" (outside clinical trials), with early deaths and patients surviving many years. Such a variability was broadened by recent introduction or improvement of several medical therapies (endocrine agents, chemotherapy, biological agents, immunotherapy, etc) in almost each of the aforesaid cancer populations. Patients with bone metastases and myeloma usually receive antiresorptive treatment (bisphosphonates or denosumab) together with main medical therapies, with the aim to reduce the risk of SRE (Skeletal Related Events, such as bone fractures, cord compression, need of radiotherapy or surgery) and to delay SRE onset. However, antiresorptive agents are associated with adverse events, including Osteonecrosis of Jaw (ONJ) that can occur in 1-15\% of cancer and myeloma patients undergoing antiresorptive therapy. The ONJ risk increases with antiresorptive treatment duration and with the time of observation (survival). Little data have been published so far about length of survival of ONJ patients, as well as survival of patients receiving antiresorptive treatments in "real life". This kind of survival data might be useful in choosing both the 
most appropriate antiresorptive therapy (drug of choice; initial planned duration; monthly vs trimestral administrations; eventual discontinuation) as well as the most effective ONJ treatment : "conservative" versus surgical treatment, the latter being restricted according to some recommendations ${ }^{[6][7]}$ but encouraged by several other authors and groups ${ }^{[8][9][10][11]}$. Herein we report the survival of ONJ cancer and myeloma patients, after the start of antiresorptive treatment, from a large regional case series. ${ }^{[12][13]}$

Materials and Methods: Along 16-years (Jan $1^{\text {st }}, 2003-31^{\text {th }}$ Dec, 2018), retrospective collection of patient characteristics, clinic and prognostic data was investigated. Medical oncology, haematology, and oral care Units of North-Western Italy (Rete Oncologica di Piemonte e Valle d'Aosta) were consulted to acquire data of ONJ patients. Acquisition of the following data was pursued: sex and age distribution, underlying cancer disease, type of drug regimen administered- zoledronate, denosumab, others - and date of death of deceased patients. Kaplan-Meier curves were deployed to obtain the actuarial median of survival after the beginning of antiresorptive treatment (bisphosphonates or denosumab), expressed in months, as well as the actuarial survival rate at two, three and four years.

Results Data from 509 patients (196 M, 313 F) were acquired. Median age was of 68 (32-90) years, with $115(22.6 \%)$ under 60 years old, 170 (33.4\%) of 60-70 years old, 224 (44\%) over 70 years old. Underlying diseases were: breast cancer (218/509, 43\%), multiple myeloma (129/509, 25.3\%), prostate (93/509, 18.3\%) lung cancer $(34 / 509,6.7 \%)$, renal cell cancer $(13 / 509,2.5 \%)$, other type of cancer $(22 / 509,4.2 \%)$.

Sex distribution for each cancer was as follows: breast cancer 5 M, $213 \mathrm{~F}$; prostate cancer: 93 M; multiple myeloma: 55 M, 74 F; lung cancer: 17 M, 17 F; renal cancer 11 M 2 F; other cancers: 15 M, 7 F. Median age (+ DS) at ONJ diagnosis for each cancer was as follows: breast cancer: $67 \pm 9.1$ (range 39-89) years; prostate cancer: $71 \pm 5.5$ (age range 55-86) years; myeloma: $72 \pm 7.4$ (range 47-89) years; lung cancers: $67 \pm 7.2$ (range: 49-80) years; renal cancers: $59 \pm 6.4$ (age range 46-73) years; others: $66 \pm 8.5$ (range 40-83) years.

Main antiresorptive treatment was as follows: zoledronic acid: $79.4 \%$; denosumab: $5.3 \%$; other drugs/sequences: $15.3 \%$.

Observed actuarial median survival after start of treatment was as follows:

- breast cancer 66 (95\% Cl: 57-77) months (Fig.1);

- prostate cancer 47.3 (95\% Cl: 37.8-51.9) months (Fig.2) ;

- multiple myeloma 77.3 (95\% Cl: 61.9-86.8) months (Fig.3);

- lung cancer 29.4 (95\% Cl: 20.4-40.1) months;

- renal cancer 39.4 (95\% Cl: 20-107.3) months;

- others 82.4 (95\% Cl: 51.9-150.9) months. 
From the actuarial survival curves the following survival rates emerged at 2, 3, 4 years, respectively:

- breast cancer : 91.7\%, 80.2\%, 67.4\%;

- myeloma: $91.4 \%, 83.7 \%, 68.9 \%$;

- prostate cancer : $78.4 \%, 61.2 \%, 41.9 \%$;

- lung cancer : 55.8\%, 32.3\%, 23.5\%;

- renal cancer : $61.5 \%, 53.8 \%, 46.1 \%$;

- other cancers: $81.8 \%, 77.3 \%, 68.1 \%$.

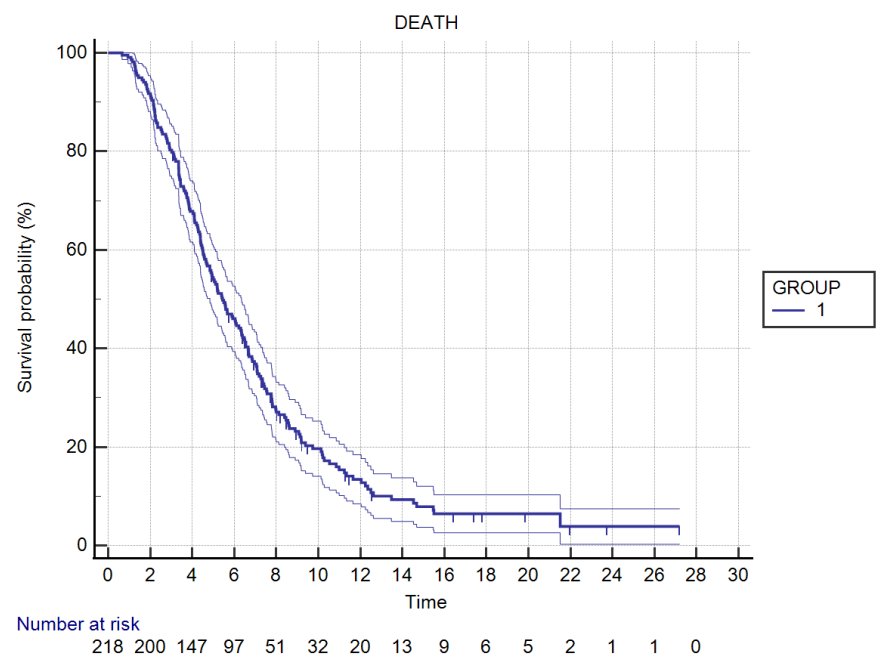

Fig. 1 Breast cancer patients: actuarial (Kaplan-Meier) survival curve

Conclusions. : In ONJ patients, expected survival after the start of antiresorptive treatment is medially long some years. The comparison of this survival with that of "real life" patients undergoing antiresorptive treatment and not developing ONJ

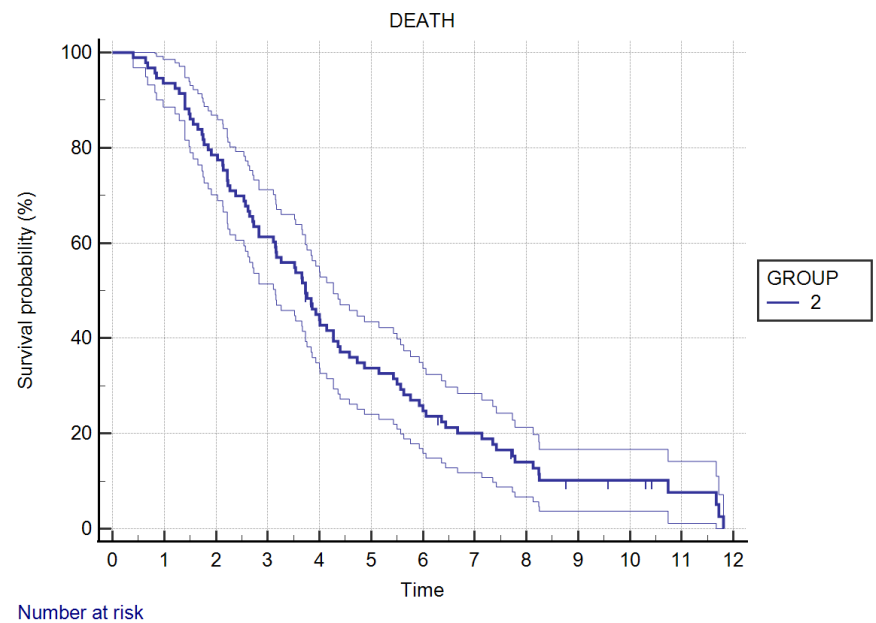
$\begin{array}{ccccccccccccc}\begin{array}{c}\text { Number at risk } \\ 93\end{array} & 87 & 73 & 57 & 39 & 30 & 22 & 17 & 11 & 7 & 6 & 3 & 0\end{array}$

Fig. 2 Prostate cancer patients: actuarial (Kaplan-Meier) survival curve

(overall, and long surviving) might be of value : a) to predict the ONJ risk among long surviving patients;

b) to establish the real ONJ risk in patients surviving more than one or two years (and to chose the best antiresorptive treatment duration); c) to evaluate the chance of surgical treatment of ONJ disease (often denied on the base of a supposed expected short survival).

\section{References}

1. ^S. D'Oronzo, S. Wood, J.E. Brown. (2021). “The use of bisphosphonates to treat skeletal

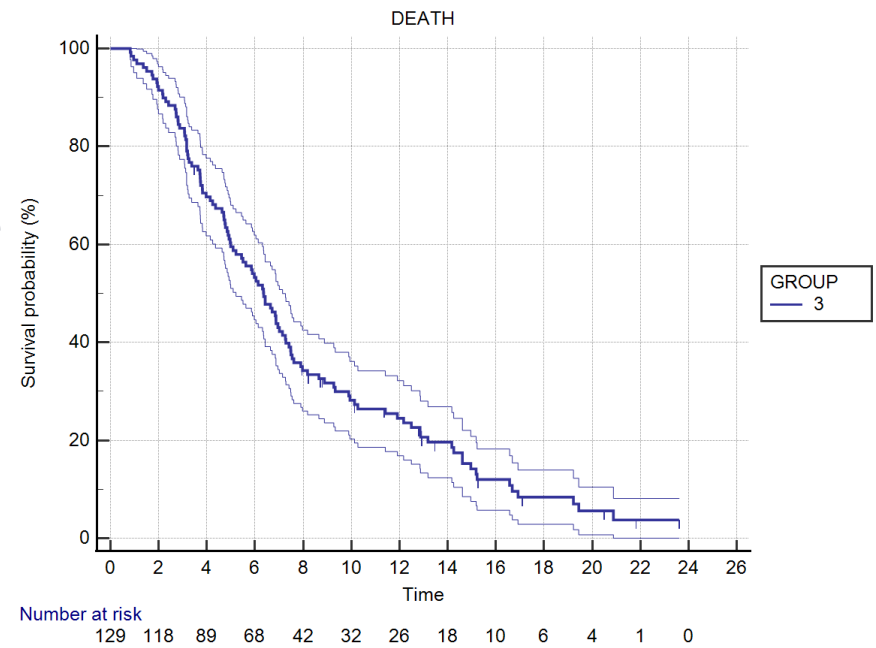

Fig. 3 Myeloma patients: actuarial (Kaplan-Meier) survival 
complications in solid tumours". Bone, vol. 147 ,

115907. doi:10.1016/j.bone.2021.115907.

2. `D. Southcott, A. Awan, K. Ghate, M. Clemons, et al. (2020). Practical Update for the Use of BoneTargeted Agents in Patients with Bone Metastases from Metastatic Breast Cancer or CastrationResistant Prostate Cancer. Current Oncology, vol. 27 (4), 220-224. doi:10.3747/co.27.6631.

3. `Suparna B. Wedam, Julia A. Beaver, Laleh Amiri-Kordestani, Erik Bloomquist, et al. (2018). US Food and Drug Administration Pooled Analysis to Assess the Impact of Bone-Only Metastatic Breast Cancer on Clinical Trial Outcomes and Radiographic Assessments. JCO, vol. 36 (12), 1225-1231. doi:10.1200/jco.2017.74.6917.

4. 'Marc Braunstein, Jonathan Weltz, Faith Davies. (2021). A new decade: novel immunotherapies on the horizon for relapsed/refractory multiple myeloma. Expert Review of Hematology. doi:10.1080/17474086.2021.1909469.

5. ^Evangelos Terpos, Elena Zamagni, Suzanne Lentzsch, Matthew T Drake, et al. (2021). Treatment of multiple myeloma-related bone disease: recommendations from the Bone Working Group of the International Myeloma Working Group. The Lancet Oncology, vol. 22 (3), e119-e130. doi:10.1016/s14702045(20)30559-3.

6. `Salvatore L. Ruggiero, Thomas B. Dodson, John Fantasia, Reginald Goodday, et al. (2014). American Association of Oral and Maxillofacial Surgeons Position Paper on Medication-Related Osteonecrosis of the Law-2014 Update. Journal of Oral and Maxillofacial Surgery, vol. 72 (10), 1938-1956. doi:10.1016/j.joms.2014.04.031.

7. ^Noam Yarom, Charles L. Shapiro, Douglas E. Peterson, Catherine H. Van Poznak, et al. (2019). Medication-Related Osteonecrosis of the Jaw: MASCC/ISOO/ASCO Clinical Practice Guideline.JCO, vol. 37 (25), 2270-2290. doi:10.1200/jco.19.01186.

8. `Alberto Bedogni, Giorgia Saia, Giordana Bettini, Anita Tronchet, et al. (2011). Long-term outcomes of surgical resection of the jaws in cancer patients with bisphosphonate-related osteonecrosis. Oral Oncology, vol. 47 (5), 420-424. doi:10.1016/j.oraloncology.2011.02.024.

9. ^Morten Schiodt, Sven Otto, Stefano Fedele, Alberto Bedogni, et al. (2019). Workshop of European task force on medication-related osteonecrosis of the jaw-Current challenges. Oral Dis, vol. 25 (7), 1815 1821. doi:10.1111/odi.13160.

10. `Sven Otto, Christoph Pautke, Tim Van den Wyngaert, Daniela Niepel, et al. (2018). Medication-related osteonecrosis of the jaw: Prevention, diagnosis and management in patients with cancer and bone metastases. Cancer Treatment Reviews, vol. 69 , 177-187. doi:10.1016/j.ctrv.2018.06.007.

11. `Giuseppina Campisi, Alberto Bedogni, Vittorio Fusco.. (2020). Raccomandazioni clinico-terapeutiche sull'osteonecrosi delle ossa mascellari (ONJ) farmaco-relata e sua prevenzione. Unipapress, Palermo (Italy).

12. 'Vittorio Fusco, Claudia Galassi, Alfredo Berruti, Cinzia Ortega, et al. (2013). Decreasing Frequency of Osteonecrosis of the Jaw in Cancer and Myeloma Patients Treated with Bisphosphonates: The 
Experience of the Oncology Network of Piedmont and Aosta Valley (North-Western Italy). ISRN Oncology, vol. 2013 , 1-8. doi:10.1155/2013/672027.

13. `V. Fusco, M. Cabras, F. Erovigni, A. Dell'Acqua, et al. (2020). A multicenter observational study on Medication-Related Osteonecrosis of the Jaw (MRONJ) in advanced cancer and myeloma patients of a cancer network in North-Western Italy. Med Oral. doi:10.4317/medoral.24318. 